\title{
The surface density of Extremely Red Objects in high-z quasar fields
}

\author{
M. Wold ${ }^{a}$ L. Armus ${ }^{b}$ G. Neugebauer ${ }^{c}$ T.H. Jarrett ${ }^{d}$ \\ M.D. Lehnert ${ }^{\mathrm{e}}$ \\ a JPL Postdoctoral Researcher, SIRTF Science Center/Caltech \\ ${ }^{\mathrm{b}}$ SIRTF Science Center/Caltech \\ ${ }^{\mathrm{c}}$ Caltech \\ d IPAC/Caltech \\ e Max-Planck-Institut für Astrophysik
}

\begin{abstract}
We report on a study of the surface density of Extremely Red Objects (EROs) in the fields of 13 radio-loud quasars at $1.8<z<3.0$ covering a total area of 61.7 $\operatorname{arcmin}^{2}$. There is a large variation in the ERO surface density from field to field, and as many as 30-40\% of the fields have roughly $4-5$ times more EROs than what is expected from a random distribution. The average surface density exceeds the value found in large random-field surveys by a factor of $2-3$, a result which is significant at the $>3 \sigma$ level. Hence, it appears that the quasar lines of sight are biassed towards regions of high ERO density. This might be caused by clusters or groups of galaxies physically associated with the quasars. However, an equally likely possibility is that the observed ERO excess is part of overdensities in the ERO population along the line of sight to the quasars. In this case, the non-randomness of quasar fields with respect to EROs may be explained in terms of gravitational lensing.
\end{abstract}

Key words: galaxies: clusters - quasars: gravitational lensing

\section{Introduction}

EROs are galaxies with very red optical to near-infrared colours. Several definitions of EROs are in use, but the among the most common are $R-K \geq 5$ and $I-K \geq 4$. The colour criterion selects $z \approx 1-2$ early-type galaxies with passively evolving stellar populations characterized by strong $4000 \AA$ breaks in their spectral energy distributions, but also dusty starbursting galaxies which 
have their UV-flux attenuated by internal dust. The first EROs were discovered in the deep near-infrared surveys by Elston, Rieke \& Rieke (하), and since then there have been several surveys aimed at studying their spectroscopic (4), photometric $(13 ; 14 ; 10)$ and clustering properties $(5 ; 12)$.

Here, we have searched for EROs in the fields of $z=1.8-3.0$ quasars. It has been suggested that the fields of high-redshift radio galaxies and quasars often have an excess of EROs $(11 ; 9 ;$; 3 ; 15; 7). E.g. Cimatti et al. (3) find an excess of $R-K \geq 6$ EROs in 14 radio galaxy and quasars fields at $1.5<z<2.0$ and Hall et al. (7) find that EROs are about 3 times more numerous in the fields of $z \approx 1.5$ quasars. Several possibilities for the ERO excess exist, but the favored explanation seems to be that the EROs are early-type galaxies associated with the quasars, perhaps signifying a forming cluster or a galaxy group at the quasar redshift. Another possibility is that the ERO excess traces foreground clusters or overdensities in the ERO population which gravitationally magnify the quasars, boosting them into flux-limited samples. In either case, the ERO excess may represent clusters in formation at cosmologically significant epochs. Care must be taken, however, because the angular distribution of EROs on the sky is characterized by overdensities and large voids (5; 12). This causes a strong field-to-field variation in the ERO surface density, especially if the fields are small.

\section{The quasar sample}

The quasar fields presented here are part of a larger ongoing survey to study the host galaxies (with the $\mathrm{NIRC}^{1}$ on Keck I) and environments of radioloud and radio-quiet quasars at $1.8<z<3.0$. The full sample consists of 40 quasars from the catalogs by Barthel et al. (1) and Hewitt \& Burbidge (8), and are nearly evenly distributed between radio-loud and radio-quiet systems with comparable $V$ magnitudes and redshifts. In order to study the environments of these quasars, we have obtained deep $R$ and $K_{s}$ images with the COSMIC ${ }^{2}$ and the PFIRCAM ${ }^{3}$ instruments on the Palomar 200 inch telescope. The $R$ band imaging is completed, and a subset of 13 radio-loud quasar fields have been imaged in $K_{s}$. The ERO samples are drawn from an area of $2.1 \times 2.1$ $\operatorname{arcmin}^{2}$ around each quasar (the region of overlap of the $R$ and $K_{s}$ imaging). The $5 \sigma$ detection limits in the $R$ - and the $K_{s}$-filters are $\approx 25$ and $\approx 20.5$, respectively, and this has allowed us to search for EROs in the quasar fields using the $R-K \geq 5$ ERO definition.

\footnotetext{
1 Near Infrared Camera

2 Carnegie Observatories Spectroscopic Multislit and Imaging Camera

3 Prime Focus Infrared Camera
} 


\section{Results}

The colour-magnitude diagram of objects detected in the quasar fields is shown in Fig. 1. In total, there are 160 objects with $R-K \geq 5.0$. Using the SEXTRACTOR (2) star-galaxy classifier, and treating all objects at $K_{s}<18$ with a star-galaxy class greater than 0.85 as stars, we find that six of the 160 EROs are possible stars. Excluding these yields a surface density of 1.26 EROs per $\operatorname{arcmin}^{2}$ at $K_{s} \leq 19$, and $2.35 \operatorname{arcmin}^{-2}$ at $K_{s} \leq 20$. This surface density is two-three times higher than what is found in wide-field random surveys, typically 0.5 at $K_{s} \leq 19$ and $1.4 \operatorname{arcmin}^{-2}$ at $K \leq 20(5 ; 12)$. Although there is a large variation in the ERO surface density from field to field, approximately 30-40\% of the quasar fields have notably large overdensities, a relatively large fraction of the sample in studies like this.

At the resolution of our images ( $\approx 1$ arcsec), it is difficult to distinguish between stars and extended objects, and SEXTRACTOR has problems with star-galaxy separation at faint magnitudes. We therefore performed the stargalaxy separation only at $K_{s}<18$. The contamination of faint red stars among EROs is uncertain, but in our case it is likely to be small since the quasar fields lie at high galactic latitudes ( $>35 \mathrm{deg}$, except three which lie between 21 and $27 \mathrm{deg})$. For completeness, we also carried out the analysis using star-galaxy separation regardless of $K_{s}$ flux. This resulted in $\approx 20 \%$ (as compared to $4 \%$ ) of the EROs being classified as stars. This of course lowers the ERO counts, but our conclusions remain unaltered. The surface densities using this approach are 1.08 at $K_{s} \leq 19$ and $1.93 \mathrm{arcmin}^{-2}$ at $K_{s} \leq 20$. We note that Mannucci et al. (10) find $\approx 10 \%$ stars at $K^{\prime}<20$ based on $R, J$ and $K^{\prime}$ imaging.

In Fig. 2, we compare the average cumulative surface density of EROs in our quasar fields with random field surveys from the literature. The field counts are represented by the surveys of Daddi et al. (5), Roche et al. (12) Cimatti et al. (4) and Scodeggio \& Silva (13), and they are seen to agree well with each other, except the counts in the Chandra Deep Field (CDF) by Scodeggio \& Silva. This discrepancy is most likely caused by the inhomogeneous distribution of EROs on the sky, given that the area of the CDF is only $43 \operatorname{arcmin}^{2}$. The counts in our quasar fields are seen to be consistently higher than the random field counts at all flux levels. This is not what we expect if we are sampling the random field ERO population with our 13 widely separated fields. The average ERO surface density from several small, widely separated fields should in principle be similar to the surface density in a large, contiguous field. Hence, it appears that the quasar fields are biassed toward regions of high ERO density.

Using the average of Daddi et al's and Roche et al's counts, we predict that our quasar fields should contain $\approx 27$ EROs at $K_{s} \leq 19$, whereas the ob- 


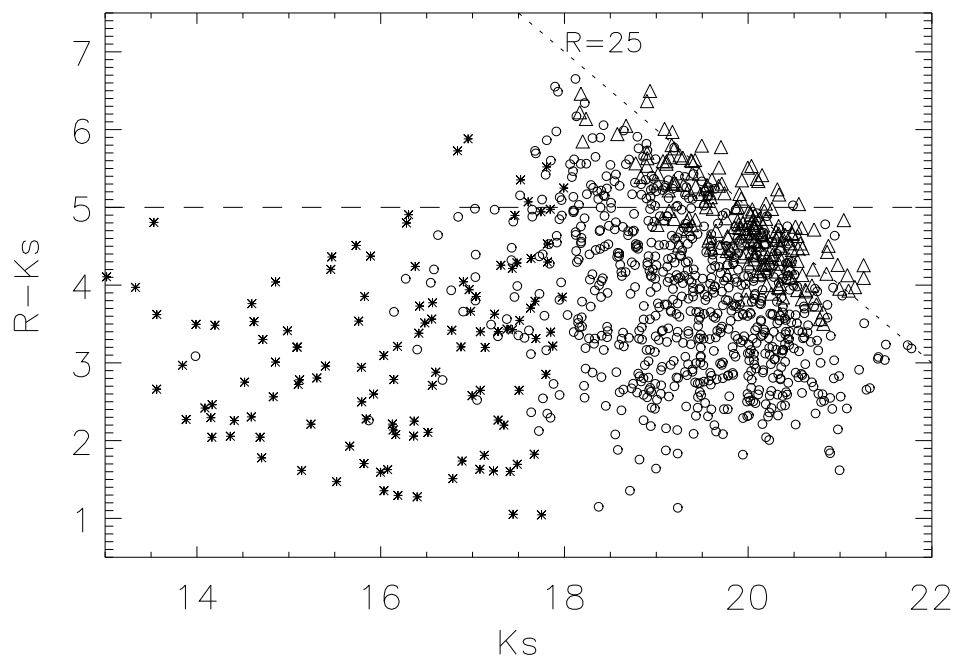

Fig. 1. Colour-magnitude diagram of objects in the 13 quasar fields. The open circles are $\geq 5 \sigma$ detections in $K_{s}$, and the triangles show objects with a detection significance $<3 \sigma$ in $R$, where a lower limit to their $R-K_{s}$ colour is given assuming a $3 \sigma$ detection in $R$. The asterisks are objects classified as stars. The horizontal line across the plot shows the ERO definition employed in this work.

served number is 79. There is thus a clear excess of EROs in the quasar fields, but given that the EROs cluster strongly and therefore that the field-to-field variation is large, is the excess we observe significant?

In order to answer this question, we calculate (a) the expected number of EROs over the total area of our fields from the literature surveys, $N_{\text {field }}$, and (b), the expected variance, $\sigma^{2}$, of the counts given that the ERO distribution is non-random, i.e. $\sigma^{2}=N_{\text {field }}\left(1+N_{\text {field }} A_{\omega} C\right)$ (5), where $C$ is a factor that depends on the area of the field and $A_{\omega}$ is the amplitude of the ERO angular two-point correlation function as found by Daddi et al. (5) and Roche et al. (12). The significance of the excess in the quasar fields is thereafter evaluated as $\left(N_{\text {qso }}-N_{\text {field }}\right) / \sigma$, where $N_{\text {qso }}$ is the observed number of EROs in the quasar fields. The result of this calculation shows that the significance of the excess

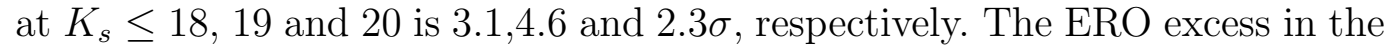
quasar fields therefore appears to be significant, even after taking into account the large fluctuations in the ERO surface density.

\section{Discussion}

Our analysis strongly suggests that the quasar lines of sight are biassed toward regions of high ERO density, and our result is consistent with that of Cimatti et al. (3) and Hall et al. (7). At the moment it is impossible to tell whether the EROs are at the same redshift as the quasars or if they are in 


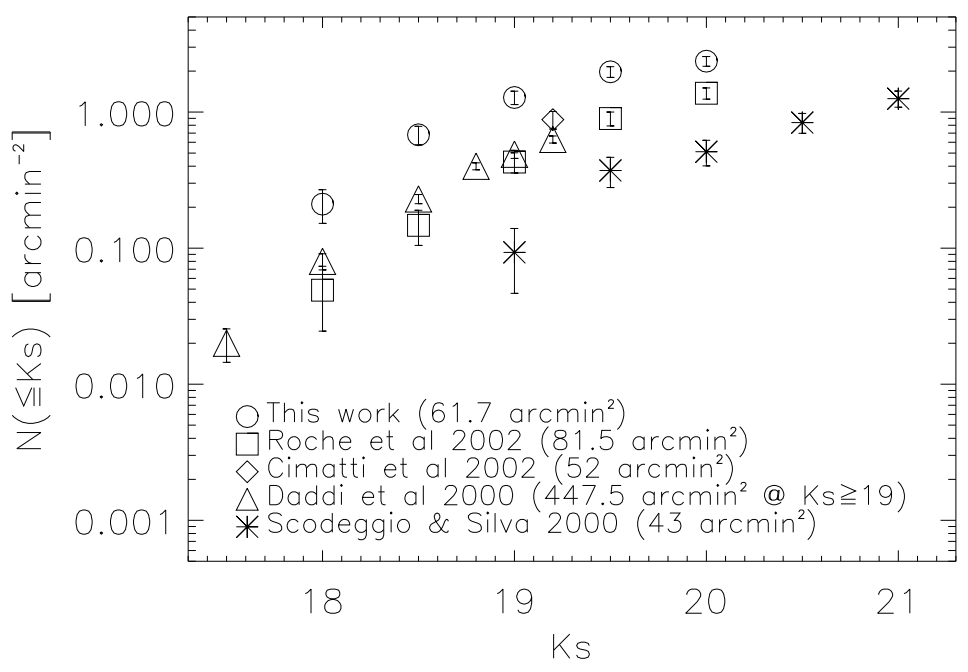

Fig. 2. Cumulative ERO surface density in our quasar fields (open circles) compared with various literature samples of EROs in random fields. Note that a comparison has only been made with surveys defining EROs as $R-K \geq 5$ or $R-K_{s} \geq 5$. The error bars are Poissonian.

the foreground. In order to address this, either photometric or spectroscopic redshifts are needed. We therefore offer two different explanations for the observed excess and discuss each of them below:

The excess might be caused by galaxies, both early types and dusty starbursts, at the quasar redshifts. In this case, a major part of the EROs could be the early-type population of forming(?) clusters or groups of galaxies physically associated with the quasars. The evidence in favor of this consists of observations finding that luminous quasars and radio galaxies often reside in regions of high galaxy density. Another possibility is that the quasars are markers of large-scale structure traced by the EROs, such as walls or sheets of galaxies, as suggested by Thompson, Aftreth \& Soifer (15). Our current $K_{s}$-band images are too small to determine whether the EROs cluster around the quasars or if they are just part of a larger-scale overdensity, but we are currently awaiting wide-field $\left(9 \times 9 \operatorname{arcmin}^{2}\right)$ near-infrared data to properly address this issue.

There is no evidence for a significant excess in the $R$ - and $K_{s}$-band counts in our fields, which we might expect if there are relatively rich clusters in the fields. However, this does not exclude the possibility that the excess is caused by clusters or groups of galaxies because most cluster members would simply be swamped in the large numbers of foreground sources. By selection on $R-K_{s}$ colour we can find the ellipticals at $z \approx 1-2$ preferentially against much of the confusing foreground galaxies.

Another interesting possibility is that the excess is caused by EROs foreground to the quasars, either associated with clusters or groups of galaxies, or just 
overdensities ('clumps') in the ERO population. In this case, lensing magnification bias is likely to be important for the quasars. By pointing the telescope to a distant quasar, one is more likely to pick out lines of sight where there are mass concentrations along the line of sight which gravitationally amplify the quasar light.

\section{$5 \quad$ Future and ongoing work}

In order to address the question of how extended the ERO excess is, we have started an imaging programme in $J$ and $K_{s}$ using the new WIRC $^{4}$ instrument on the Palomar 200 inch. The WIRC has a field of view of $9 \times 9 \operatorname{arcmin}^{2}$ which matches the size of our wide-field $R$-band images. We are also planning to obtain spectra and morphologies of the EROs. This will allow us to determine redshifts, spectral types and structural parameters of the EROs, thereby opening up for an investigation of the Fundamental Plane of early-type galaxies at cosmologically significant epochs.

\section{References}

[1] Barthel P.D., Miley G.K., Schilizzi R.T., Lonsdale C.J., A $\& A S 73$ (1988) 515.

[2] Bertin E., Arnouts S., A $\& A S 117$ (1996) 393.

[3] Cimatti A., Villani D., Pozzetti L., di Serego Alighieri S., MNRAS 318 (2000) 453.

[4] Cimatti A., et al., A\&A 381 (2002) L68.

[5] Daddi R., Cimatti A., Pozzetti L., Hoekstra H., Röttgering H.J.A., Renzini A., Zamorani G., Mannucci F., A $\mathscr{G A} 361$ (2000) 535.

[6] Elston R., Rieke M.J., Rieke G.H., ApJ 341 (1988) 80.

[7] Hall P.B., et al., AJ 121 (2001) 1840.

[8] Hewitt A., Burbidge G., ApJS 87 (1993) 451.

[9] Hu E.M., Ridgway S.E., AJ 107 (1994) 1303.

[10] Mannucci F., et al., MNRAS 329 (2002) L57.

[11] McCarthy P.J., Persson S.E., West S.C., ApJ 386 (1992) 52.

[12] Roche N.D., Almaini O., Dunlop J., Ivison R.J., Willott C.J., MNRAS 337 (2002) 1282.

[13] Scodeggio M., Silva D.R., A 64359 (2000) 953.

[14] Smail I., Owen F.N., Morrison G.E., Keel W.C., Ivison R.J., Ledlow M.J., ApJ 581 (2002) 844.

[15] Thompson D., Aftreth O., Soifer B.T., ApJ 120 (2000) 2331.

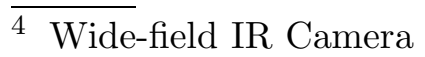

